\title{
Peer Review, Impact Factor und Leserpräferenz geben Hinweise zur Erstellung von Publikationen für die klinische Behandlung
}

\section{Peer Review and Impact Factor as well as Preferential Reading Give Hint to Authors of Papers for Clinical Issues}

\section{Hintergrund}

\section{$\nabla$}

Für die Beurteilung von wissenschaftlichen Aktivitäten stehen verschiedene bibliometrische Messverfahren zur Verfügung [3,15, 19,24,29]. Von diesen ist als erster der Impact Factor Anfang der 1980iger-Jahre entwickelt worden und wird weltweit eingesetzt [3]; ungefähr 23000 Wissenschaftsjournale aller Forschungsrichtungen werden durch ISI Web of Science evaluiert [40].

Allgemein gewinnen diese Bewertungen trotz ihres vorwiegend zählenden Charakters ihre Wichtigkeit aus der Tatsache, dass sie z.B. bei der Bewilligung von Fördermitteln für wissenschaftliche Fragestellungen, der Verleihung von akademischen Auszeichnungen für herausragende Forschungsergebnisse oder bei der Vergabe von beruflichen Positionen im akademischen Bereich beachtet werden [11,24, 28, 29].

Aus diesen Verwendungen leitet sich seitens der Autoren das Interesse $a b$, in einer wissenschaftlichen Zeitschrift mit guter Bewertung zu publizieren. Seitens der Verlage und der Herausgeber wird verständlich, durch die sorgfältige Prozessierung der eingereichten Manuskripte diese Erwartungen zu erfüllen. Als ein Ergebnis dieser gemeinsamen Anstrengungen ist festzustellen, dass von Jahr zu Jahr die Impact Factors für die international führenden Journale ansteigen und parallel dazu die Zahl der Zeitschriften mit Impact Factor zunimmt [9, s. S. 43-50]. Da nicht alle Arbeiten zitiert werden, sind diejenigen von besonderer Bedeutung, auf die häufiger Bezug genommen wird $[10,17]$.

Korrespondenzadressen

Prof. Dr. Ulrich Göbel ESPED-Geschäftsstelle Koordinierungszentrum für Klinische Studien Universitätsklinikum Heinrich-Heine-Universität Düsseldorf

Moorenstraße 5 40225 Düsseldorf goebelu@arcor.de

\section{Prof. Dr. Ludwig Gortner}

Klinik für Allgemeine Pädiatrie und Neonatologie Kliniken Kinderund Jugendmedizin Universitätsklinikum des Saarlandes Gebäude 9 66421 Homburg/Saar neonat@uniklinikum-saarland.de $[6,7,8,10]$. Gleichzeitig wurde mit diesen Editorials die schrittweise erfolgte Implementierung des Internet bei der Editierung der Klinischen Pädiatrie - Clinical Research und Practice in Pediatrics vorbereitet und auf die hierdurch induzierten Effekte hingewiesen. So sind bei dieser 1880 gegründeten Zeitschrift kontinuierlich Neuerungen zu bahnen und durch die Autoren umzusetzen. Wichtige Impulse können dabei von den Lesern aufgrund ihrer Präferenzen und von den Autoren durch ihre Zitierungen abgeleitet werden.

Als Folge der vielfältigen und raschen Fortschritte in der Medizin wachsen die Ansprüchen an die verfügbaren Ressourcen, die schon immer als knapp angesehen wurden und nicht entsprechend dem Wissensfortschritt vermehrt bereitgestellt werden. Deshalb gilt es, auch die Umsetzung von neuen und auf Evidenz basierenden Behandlungsempfehlungen im klinischen Alltag zu unterstützen sowie bei der Optimierung der interdisziplinären Zusammenarbeit aus wissenschaftlicher Sicht mitzuwirken. Ziele sind die Verbesserung der Behandlungsqualität und die Schonung der Ressourcen.

\section{Einreichung und Begutachtung von Manuskripten \\ $\nabla$}

Im Dezember 2008 ist für die Einreichung und Prozessierung der Manuskripte die professionelle Unterstützung mittels ScholarOne Manuscripts eingeführt worden [34]. Die Manuskripte werden doppelt verdeckt begutachtet, sodass die Gutachter nicht wissen, von wem und woher das Manuskript stammt, und der Autor erfährt nicht die Namen der beiden Gutachter [42]. Das Verfahren ist nach einer einjährigen Kennenlernphase ausführlich dargestellt worden [8]. Wurden im Jahr 2009 durchschnittlich 9,3 Manuskripte pro Monat eingereicht, so ist die Zahl im Jahr 2011 auf 11,8 pro Monat angestiegen. Überwiegend handelt es sich um Originalarbeiten, die auch die ausführlichen Falldarstellungen mit Erweiterung der Kenntnisse zum Krankheitsbild entsprechend den revidierten Autorenhinweisen von 2008 beinhalten [6] ( 0 Abb. 1).

Inzwischen sind über ScholarOne Manuscripts mehr als 400 Manuskripte eingereicht worden, sodass recht valide Informationen verfügbar sind. Die Zeitspanne zwischen Einreichung, Begutachtung durch externe Gutachter, Revision

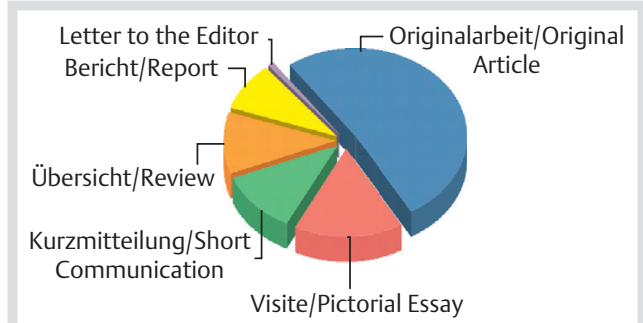

Abb. 1 Art und Zahl der 2011 für die Klinische Pädiatrie eingereichten Manuskripte.

141 Neueinreichungen; durchschnittlich 11,8 Manuskripte pro Monat. 
der Manuskripte durch die Autoren und erneute Begutachtung bis zur Absendung der endgültigen Entscheidung hinsichtlich Ablehnung oder Annahme durch den Managing Editor hat sich im Median von 61 Tagen in 2009 auf 51 Tage in 2011 weiter verkürzt. Die durchschnittliche Bearbeitungsdauer von 78 Tagen ist durch einige wenige Manuskripte bedingt, die mehr als 2-mal revidiert wurden und deshalb lange Zeit bis zur endgültigen Entscheidung benötigten; beide Kennzahlen sind im Vergleich zu anderen Zeitschriften des Thieme Verlags kurz und belegen gleichermaßen die rasche internetbasierte Prozessierung der Manuskripte mittels ScholarOne Manuscripts wie die zügige Tätigkeit der von den Fachgesellschaften der Kinder- und Jugendmedizin benannten Gutachter. Etwa ein Drittel der Manuskripte erreichte bei der doppelt verdeckten Begutachtung nicht die für eine Publikation ausreichende Priorität.

\section{Zunahme der Veröffentlichungen pro Jahr}

Bei der insgesamt größeren Zahl von eingereichten Manuskripten konnte trotz der limitierten Zahl verfügbarer Druckseiten die Ablehnungsrate auf diesem Niveau gehalten werden. So haben die 2008 revidierten Autorenhinweise zu kürzeren Manuskripten geführt. Weiterhin werden Tabellen und Abbildungen, die für die vollständige Darstellung der Methodik oder Ergebnisse wichtig, aber nicht für das direkte Verständnis der Arbeit notwendig sind, nur online veröffentlicht. Da diese Maßnahmen jedoch nicht ausreichten, gibt der Thieme Verlag seit 2009 ein zusätzliches Heft 7 heraus, um den zügigen Druck der angenommenen und elektronisch vorab veröffentlichten Manuskripte zu gewährleisten.

Die Zeitspanne zwischen der Einreichung der Manuskripte und ihrem Abdruck in einem der Hefte schwankt zwischen 3 und 12

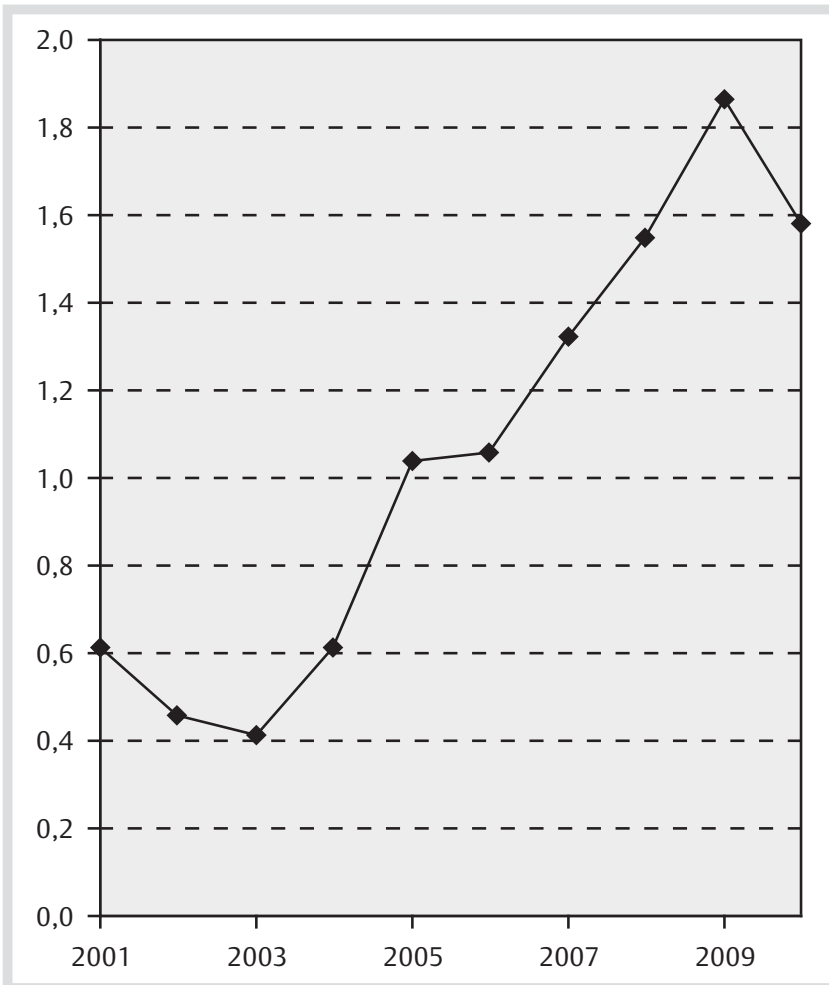

Abb. 2 Die Klinische Pädiatrie: Impact Factor 2001-2010.

Aktueller Impact Factor 2010: 1,583.
Monaten und ist wesentlich von der Subspezialität und dem Begutachtungsverlauf abhängig. 2 der 7 verfügbaren Hefte (Heft 3 für Mai/Juni und Heft 6 für November) werden im Auftrag der Gesellschaft für Pädiatrische Onkologie und Hämatologie $(\mathrm{GPOH})$ erstellt und verfügen über deutlich mehr Druckseiten (75 anstelle von 48). Da für diese Hefte feste Einreichungszeiten festgelegt sind (15.01. und 15.07.), sind die Publikationszeiten für diese Arbeiten besonders kurz. Insgesamt haben diese Maßnahmen dazu geführt, dass sich die Zahl der Veröffentlichungen - ohne Berücksichtigung von Supplementen und Abstracts - von 60 in 2005 auf 99 in 2011 erheblich erhöht hat.

\section{Impact Factor und Ranking für die Jahre 2001 bis 2010} $\nabla$

Der Impact Factor ist ein Quotient, der z. B. für das Jahr 2010 aus der Zahl der Zitierungen von Arbeiten der Klinischen Pädiatrie der Jahre 2008 und 2009 (Nominator) sowie der Gesamtzahl der in 2008 und 2009 publizierten Manuskripte (Denominator) gebildet wird [3,34]. Dieser Wert ist für die Jahre 2003-2009 kontinuierlich gestiegen ( $\bullet$ Abb. 2). Die für das Jahr $2010 \mathrm{zu}$ verzeichnende Abnahme des Impact Factors auf 1,583 ist wesentlich auf die starke Zunahme von publizierten Manuskripten zurückzuführen, da sich hierdurch der Denominator vergrößert hat, aber der Nominator nicht entsprechend mitgewachsen ist. Als Folge hat sich auch die Positionierung der Klinischen Pädiatrie im internationalen Vergleich mit den anderen Zeitschriften der Liste Pediatrics nachteilig verändert und nimmt jetzt Rang 49 unter den 107 gelisteten Zeitschriften mit Impact Factor ein ( $\bullet$ Abb. 3). Hierbei ist zu berücksichtigen, dass allein für den deutschsprachigen Raum von 10 Verlagen insgesamt 14 Zeitschriften auf dem Gebiet der Kinder- und Jugendmedizin herausgegeben werden $[1,4,5,13,14,20,23,25,27,30,33,35-37]$, von denen aber nur 2 Journale über einen Impact Factor verfügen $[4,35]$.

Aufgrund des seit 2003 kontinuierlich angestiegenen Impact Factors rangiert die Klinische Pädiatrie seit 2007 in der vorderen Hälfte der gelisteten Journale der Liste Pediatrics, die parallel dazu von 68 auf 110 Zeitschriften kontinuierlich gewachsen ist ( $\bullet$ Abb. 3). Auch in dieser Zunahme der gelisteten Zeitschriften ist ein Grund für den allgemein zu verzeichnenden Anstieg des Impact Factors zu sehen, da nur diese für die Ermittlung heran-

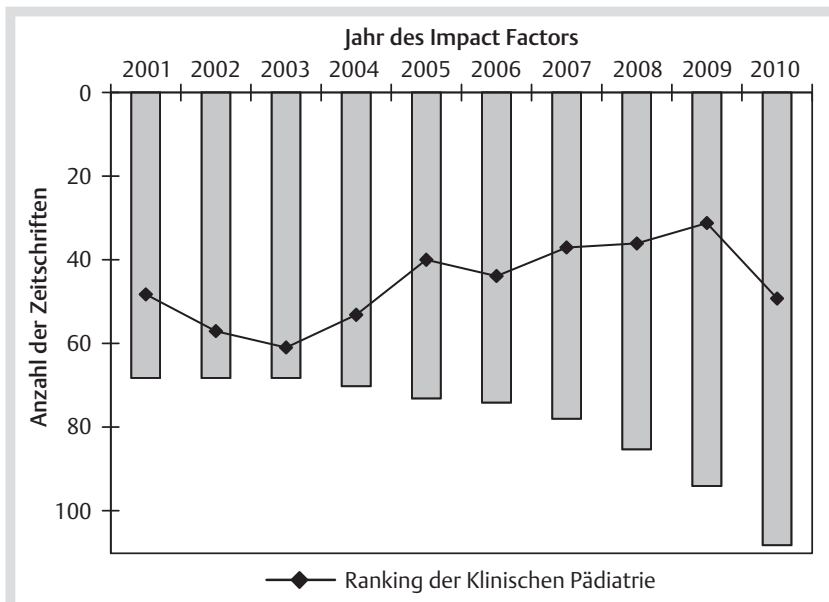

Abb. 3 Entwicklung des Rankings der Klinischen Pädiatrie innerhalb der Fachliste Pediatrics der Journal Citation Reports. Die Klinische Pädiatrie belegt derzeit Platz 49 von 108 (110) gelisteten Pädiatriezeitschriften. 
gezogen werden. Infolgedessen besteht ein statistisch auffälliger Trend zwischen der Zahl der Journale einer Fachliste und der Höhe des Impact Factors der auf Rang 1 und 2 gelisteten Zeitschriften [9, s. S. 43-50]. Für die Klinische Pädiatrie ist die Relevanz des allgemein zu verzeichnenden Anstiegs des Impact Factors auch daran abzulesen, dass der Wert 1.564 in 2008 noch für Rang 36 unter den damals 86 gelisteten Zeitschriften qualifizierte [39] ( $\odot$ Abb. 3).

Da die Klinische Pädiatrie mit den jährlich erscheinenden 2 Heften im Auftrag der GPOH über einen ausgewiesenen Schwerpunkt verfügt, ist der Vergleich sowohl mit den allgemeinpädiatrischen Zeitschriften wie mit den pädiatrischen Fachzeitschriften für Onkologie, Hämatologie, Immunologie und Hämostaseologie sinnvoll. Bei beiden Vergleichen nimmt die Klinische Pädiatrie innerhalb der Fachliste Pediatrics unter den international ausgerichteten Journalen einen beachtlichen Mittelfeldplatz ein. Dagegen haben die Journale mit dem Namen eines Landes im Titel überwiegend einen Rang im hinteren Drittel [39]. Generell werden die Publikationen mit einer umschriebenen Ortsbezeichnung in der Überschrift seltener zitiert als vergleichbare Arbeiten ohne diese Spezifizierung [17].

Weiterhin ist zu berücksichtigen, dass besonders gute und wichtige Forschungsergebnisse bevorzugt in Journalen mit besonders hohem Impact Factor und ohne Altersbegrenzung der Patienten publiziert werden, sodass diese bevorzugt zitierten Arbeiten den pädiatrischen Zeitschriften entgehen. Beispielhaft sind die Publikationen zu den Ergebnissen der prospektiven interdisziplinären und multizentrischen Therapieoptimierungsstudien der GPOH zu nennen, die vor 2000 noch häufig in deutscher Sprache als Verhandlungsbericht in der Klinischen Pädiatrie veröffentlicht wurden; dies entfällt heute, um den Anschein einer Doppelpublikation zu vermeiden. Hinzu kommt, dass allein schon altersbedingt die pädiatrischen Patientenkollektive erheblich kleiner und dadurch prospektive Studien selbst auf nationaler Basis oft erschwert oder gar unmöglich sind.

Diese Gegebenheiten tragen mit dazu bei, dass die Zeitschriften der pädiatrischen Subspezialitäten in der Liste Pediatrics meist einen guten Rang haben, nicht aber in den entsprechenden Fachlisten für alle Altersgruppen [39]. Hieraus können z.B. Nachteile bei der Bewilligung von Fördermitteln erwachsen, wenn die Liste Pediatrics nicht zur Grundlage genommen wird. Aus diesen Überlegungen werden 2 Aspekte deutlich:
- Das Ranking in einer Fachliste reflektiert realistischer die Forschungsleistung als ein isoliert betrachteter hoher Impact Factor.

- Wird eine Zeitschrift in mehr als einer Fachliste geführt, kann ein Konflikt entstehen, da unterschiedliche Rangplätze für die Bewertung der Forschungsleistung herangezogen werden können.

\section{Zitierungen der Klinischen Pädiatrie in 2010 \\ $\nabla$}

Der jährlich erscheinende Journal Citation Report stellt weitere wichtige Informationen zur Verfügung, die über die einfache Mitteilung des Impact Factors für das zurückliegende Jahr und das Ranking hinausgehen [39]. So können weitere spezifische Daten für ein Journal wie für eine ganze Spezialität oder Subspezialität abgerufen oder auch Links zu Datenbanken wie z.B. von Current Contents hergestellt werden. Dies ermöglicht sowohl Rückschlüsse auf das Verhalten der Leser allgemein als auch speziell auf das der Autoren unter den Lesern einer Zeitschrift.

Zuerst ein Blick auf die Autoren. In 2010 sind 820-mal Manuskripte der Klinischen Pädiatrie in 243 verschiedenen Journalen zitiert worden ( $\bullet$ Tab. 1). Bei den TOP 10 der zitierenden Zeitschriften führt erwartungsgemäß die Klinische Pädiatrie mit 200 Zitaten, wodurch der Vernetzungsgrad innerhalb der deutschsprachigen Kinder- und Jugendmedizin reflektiert wird. Die 620 anderen Zitate verteilen sich somit auf 242 Zeitschriften, die nach fachlichen Gesichtspunkten mehrheitlich nur außerhalb der Liste Pediatrics mit ihren 110 Journalen einzuordnen sind. Von den TOP 10 zitierenden Journalen verfügen 5 über einen hohen Impact Factor $(>5)$, sodass sie den führenden Zeitschriften der entsprechenden Fachlisten zuzurechnen sind. Hierbei imponiert die Wichtung der onkologischen und hämatologischen Zeitschriften speziell für die Jahre vor 2001, als in der Klinischen Pädiatrie noch regelmäßig die Ergebnisse der multizentrischen Therapieoptimierungsstudien als Verhandlungsberichte mitgeteilt wurden.

In Summe sind knapp 40\% der in 2010 erfolgten Zitierungen der Klinischen Pädiatrie in nur 11 Zeitschriften und 60\% in 232 anderen Journalen erfolgt. Dieser letzte Aspekt ist als ein besonders positiver Effekt der Suchmaschinen zu werten und weist auf die Wichtigkeit einer informativen Überschrift hin [17]. Unter zeitlichen Aspekten ist anzumerken, dass 25 der Zitierungen schon in 2010, also in dem Jahr ihrer Veröffentlichung

Tab. 1 Anzahl der Zitierungen in 2010 von Artikeln der Klinischen Pädiatrie unter Berücksichtigung des Erscheinungsjahres und der Zeitschrift Journal Citation Reports ${ }^{\circledR}$ Science Edition (Thomson Reuters, 2010) [39].

\begin{tabular}{|c|c|c|c|c|c|c|c|}
\hline zitierende Journale & Zitate & 2010 & 2009 & 2008 & 2007-2001 & vor 2001 & \\
\hline alle: $n=243$ & 820 & 25 & 112 & 108 & 302 & 273 & \\
\hline TOP 10 Journale & & & & & & & Impact Factor \\
\hline Klin Padiatr & 200 & 13 & 78 & 69 & 29 & 11 & 1,583 \\
\hline Ped Blood Cancer & 28 & 0 & 2 & 2 & 13 & 11 & 1,948 \\
\hline Leukemia & 18 & 0 & 0 & 0 & 3 & 15 & 8,966 \\
\hline Bundesgesheitsbl & 13 & 0 & 0 & 0 & 11 & 2 & 0,595 \\
\hline Brit J Haematol & 13 & 0 & 0 & 1 & 6 & 6 & 4,942 \\
\hline Cochr DB Syst Rev & 13 & 1 & 0 & 0 & 4 & 8 & 6,186 \\
\hline J Clin Oncol & 10 & 0 & 0 & 1 & 2 & 7 & 8,970 \\
\hline Monatsschr Kinderheilkd & 10 & 2 & 1 & 2 & 5 & 0 & 0,231 \\
\hline Blood & 9 & 0 & 0 & 0 & 3 & 6 & 10,558 \\
\hline Nat Rev Endocrin & 7 & 0 & 0 & 0 & 6 & 1 & 9,191 \\
\hline Expert Rev Neuroth & 7 & 0 & 0 & 0 & 6 & 1 & folgt in $1 \mathrm{Jahr}$ \\
\hline
\end{tabular}


Tab. 2 Liste der Veröffentlichungen aus 2010 mit der größten Zahl an Herunterladungen im Volltext (Stichtag 13.07.2011)

\begin{tabular}{|c|c|c|c|c|}
\hline & Titel der Veröffentlichung & Format & Herunterladungen & Zitat \\
\hline 1 & Die PAN-Studie - der erste Schritt in die Zukunft & Editorial & 480 & {$[26]$} \\
\hline 2 & $\begin{array}{l}\text { Prävalenz angeborener Herzfehler bei Neugeborenen in Deutschland: Ergebnisse des } \\
\text { ersten Geburtsjahrganges (Juli } 2006 \text { bis Juni 2007) }\end{array}$ & Report & 463 & {$[22]$} \\
\hline 3 & $\begin{array}{l}\text { Surfactant ohne Intubation bei Frühgeborenen mit Atemnotsyndrom: Erste } \\
\text { multizentrische Daten }\end{array}$ & $\begin{array}{l}\text { Rapid } \\
\text { Communication }\end{array}$ & 316 & [21] \\
\hline 4 & $\begin{array}{l}\text { Intensivmedizinisches Reizumfeld und Stressoren - welchen Einfluss haben sie auf die } \\
\text { Gehirnentwicklung frühgeborener Kinder? }\end{array}$ & Übersicht & 164 & [16] \\
\hline 5 & $\begin{array}{l}\text { Probiotika zur Prävention der nekrotisierenden Enterokolitis - eine aktualisierte } \\
\text { Metaanalyse }\end{array}$ & Übersicht & 157 & [12] \\
\hline 6 & $\begin{array}{l}\text { Entwicklungsbedingte Koordinationsstörungen: Zum Zusammenhang von } \\
\text { motorischen und kognitiven Defiziten }\end{array}$ & Originalarbeit & 125 & {$[18]$} \\
\hline 7 & $\begin{array}{l}\text { Angeborenen Störungen der Thrombozytenfunktion in der pädiatrisch-klinischen } \\
\text { Praxis - eine diagnostische Herausforderung }\end{array}$ & Report & 120 & [38] \\
\hline 8 & Vergleich von Sufentanil vs. Fentanyl bei reifen, beatmeten Neugeborenen & Originalarbeit & 104 & [32] \\
\hline 9 & $\begin{array}{l}\text { Katzenkratzkrankheit - heterogen in der Klinischen Präsentation: } 5 \text { ungewöhnliche } \\
\text { Fälle einer Infektion mit Bartonella henselae }\end{array}$ & Originalarbeit & 102 & {$[41]$} \\
\hline 10 & $\begin{array}{l}\text { Diagnostische und therapeutische Aspekte bei angeborenen } \\
\text { Thrombozytenfunktionsstörungen im Kindesalter }\end{array}$ & Report & 101 & [31] \\
\hline
\end{tabular}

erfolgten. Da die Hälfte dieser Zitate in anderen Zeitschriften stehen, belegt dies die heute möglichen kurzen Publikationszeiten infolge der professionellen Unterstützung durch ScholarOne Manuscripts und die elektronischen Vorabveröffentlichungen. Auf Arbeiten der Jahre 2008 und 2009 entfallen 220 Zitierungen, die zu dem Impact Factor von 1,583 geführt haben. Dagegen beziehen sich insgesamt 302 Zitierungen auf die Jahre 2001-2007 und 273 auf die Zeit vor 2001. Hierdurch wird die Nachhaltigkeit von wichtigen Arbeiten in der Klinischen Pädiatrie belegt.

\section{Leserinteresse der Klinischen Pädiatrie

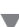

Die Klinische Pädiatrie verzichtet nahezu vollständig auf fremde Anzeigen und gehört damit zu den hochpreisigen Zeitschriften. Daher wird sie nahezu ausschließlich von Institutionen oder Bibliotheken - entweder nur online oder zusätzlich auch im Druck bezogen. Dagegen werden die Schwerpunkthefte für Onkologie und Hämatologie in größerer Zahl gedruckt und allen GPOHMitgliedern auf dem Postweg zugestellt. Das Leseverhalten der GPOH-Mitglieder kann somit nicht nachvollzogen werden, wohl aber das der Online-Leser. Für die Online-Ausgabe kann genau festgestellt werden, wie oft eine bestimmte Arbeit im Internet aufgerufen, das zugehörige Abstract geöffnet und der Volltext der Arbeit heruntergeladen werden. Von besonderem Interesse ist dabei das Verhältnis zwischen der Öffnung eines Abstracts und dem Herunterladen des Volltextes, das sich von 1:5 im Jahr 2009 auf 1:3 in 2010 verbessert hat. Öfter ist vermutet worden, dass der freie Zugang nicht nur zu Abstract, sondern zu dem ganzen Manuskript vorteilhaft für die Häufigkeit von Zitierungen sei, was sich aber in einer prospektiv angelegten Studie nicht bestätigen ließ [2].

Die 10 Artikel, die am häufigsten im Volltext heruntergeladen wurden, gehören unterschiedlichen Formaten an ( $\bullet$ Tab. 2). Originalarbeiten, die charakteristischerweise forschungsorientiert sind, führen vor den Reports/Berichten zur Optimierung der klinischen Versorgung und den Übersichtsarbeiten. Die Rapid Communications sind gleichfalls den Originalarbeiten zuzurechnen, allerdings erfüllt die hier genannte Publikation in hochgradiger Weise auch die Belange einer optimierten klinischen Behandlung. Dieses hier erkennbare Leserinteresse gilt es bei der
Weiterentwicklung der Zeitschrift möglichst zu berücksichtigen.

\section{Zusammenfassung und Schlussfolgerungen $\nabla$}

Die elektronische Einreichung von Manuskripten und ihre Prozessierung sowie Vorabveröffentlichung im Internet haben entscheidenden Einfluss auf die Gestaltung von medizinischen Fachzeitschriften genommen und ermöglichen entsprechend den raschen Entwicklungen in der Medizin eine schnelle Verfügbarkeit der Ergebnisse in Schriftform. Dies wirkt sich auf das Lese- und Zitierverhalten aus; so sind in 2010 Arbeiten der Klinischen Pädiatrie 820-mal in 243 der weltweit führenden Zeitschriften zitiert worden, die aktuell für alle Forschungsrichtungen, einschließl. der Medizin in der Journal Summary List aufgeführt sind [40]. Aus dieser Interdisziplinarität der zitierenden Autoren wird der Wert informativer Überschriften ersichtlich.

Die Zuhilfenahme eines professionellen Providers hat die Begutachtungsdauer signifikant verkürzt und durch das formalisierte Verfahren die Qualität der Gutachten auf hohem Niveau standardisiert. Hierzu trägt in besonderer Weise die doppelt verdeckte Begutachtung bei. Somit stehen der aufwendigeren Manuskripteinreichung erhebliche Vorteile gegenüber.

Seit 2004 nehmen in der Fachliste Pediatrics die gelisteten Journale kontinuierlich zu und der Impact Factor für die gelisteten Zeitschriften steigt an ohne jedoch mit anderen Fächern Schritt zu halten. Dies ist allein schon durch die Altersbegrenzung und die erheblich geringeren Patientenzahlen in plausibler Weise erklärbar. Daher verdient das Ranking einer Zeitschrift in der entsprechenden Fachliste eine höhere Beachtung als die isolierte Wertung eines Impact Factors, um die wissenschaftliche Leistung sachgerecht einzuordnen. Die Klinische Pädiatrie nimmt seit 2007 einen Rang in der vorderen Hälfte der Liste Pediatrics ein. Bei den Lesern der Klinischen Pädiatrie besteht gleichermaßen das Interesse an aktuellen wissenschaftlichen Ergebnissen wie an Informationen zur Optimierung der klinischen Versorgung. Dies ist im Kontext mit den gesetzlichen Änderungen zu sehen, die das Gesundheitssystem nachhaltig verändern. Deshalb soll zukünftig dem Bereich Clinical Practice mit den Formaten Picto- 
rial Essay/Visite, Reports/Berichte und Short Communications/ Kurzmitteilungen noch mehr Aufmerksamkeit zukommen, ohne deshalb die Belange des Bereichs Clinical Research mit den Formaten Reviews/Übersichten, Original Articles/Originalarbeiten und Case Reports/Fallberichten zu schmälern. Diese besondere Akzentverstärkung wird auf Grundlage der 2008 revidierten Autorenhinweise erfolgen [6].

Interessenkonflikt: Die Autoren erklären hiermit, dass kein Interessenkonflikt besteht.

\section{Literatur}

1 biomedpark Medien GmbH. Pädiatrix - Das Magazin für Kinderheilkunde. http://www.pädiatrix.de/home.php

2 Davis PM, Lewenstein BV, Simon DH et al. Open access publishing, article downloads, and citations: randomised controlled trial. BMJ 2008; 337: a568 doi: 10.1136/bmj.a568

3 Garfield E. Which medical journals have the greatest impact? Ann Intern Med 1986; 105: 313-320

4 Georg Thieme Verlag. Klin Padiatr 2011; 223: 1-405

5 Georg Thieme Verlag. Padiatrie up2date 2011; 6: 1-431

6 Göbel U, Gortner L. Clinical Research and Practice in Pediatrics. Klin Padiatr 2008; 220: 2-4

7 Göbel U, Gortner L. Die Klinische Pädiatrie zu Beginn des neuen Jahrzehnts. Klin Padiatr 2010; 222: 53-55

8 Göbel U, Gortner L. Die Entwicklung der Klinischen Pädiatrie seit Einführung des Online-Redaktionssystems und die DoppelblindBegutachtung von Manuskripten. Klin Padiatr 2010; 222: 231-233

9 Göbel $U$, Niem V. Rating und Ranking medizinischer Zeitschriften: Randomisiert kontrollierte Evaluation von Impact Factor und Zahl der gelisteten Journale. Klin Padiatr 2012; 224: 43-50

10 Göbel U, Sitzmann FC. Impact Factor 2006: 1,055 - die Klinische Pädiatrie im Spannungsfeld zwischen klinischer Forschung und Fortbildung. Klin Padiatr 2007; 219: 249-250

11 Grzybowski $A$. The journal impact factor: how to interpret its true value and importance. Med Sci Monit; 2009; 15: SR1-SR4

12 Guthmann F, Kluthe C, Bührer C. Probiotics for prevention of necrotising enterocolitis: An updated meta-analysis. Klin Padiatr 2010; 222: 284-290

13 Hansisches Verlagskontor GmbH. Kinder- und Jugendarzt 2011; 42: 1-598

14 Hans Marseille Verlag. pädiatrische praxis 2011; 77: 1-768

15 Hirsch JE. An index to quantify an individual's scientific research output. Proc Natl Acad Sci USA 2005; 102: 16569-16572

16 Huppertz-Kessler CJ, Verveur D, Pöschl J. Intensivmedizinisches Reizumfeld und Stressoren - welchen Einfluss haben sie auf die Gehirnentwicklung frühgeborener Kinder? Klin Padiatr 2010; 222: 236-242

17 Jacques TS, Sebire NJ. The impact of article titles on citation hits: an analysis of general and specialist medical journals. J R Soc Med Sh Rep 2010; 1: 2 doi: 10.1258/shorts.2009.100020
18 Kastner J, Petermann F. Entwicklungsbedingte Koordinationsstörungen: Zum Zusammenhang von motorischen und kognitiven Defiziten. Klin Padiatr 2010; 222: 26-34

19 Kellner AW, Ponciano LC. H-index in the Brazilian Academy of Sciences: comments and concerns. An Acad Bras Cienc 2008; 80: 771-781

20 Kirchheim + Co GmbH. Kinderärztliche Praxis 2011; 83: 1-402

21 Kribs A, Härtel C, Kattner E et al. Surfactant without intubation in preterm infants with respiratory distress: First multi-center data. Klin Padiatr 2010; 222: 13-17

22 Lindinger A, Schwedler G, Hense H-W. Prevalence of Congenital Heart Defects in Newborns in Germany: Results of the First Registration Year of the PAN Study (July 2006 to June 2007). Klin Padiatr 2010; 222: $321-326$

23 Medical Tribune Verlagsgesellschaft mbh, Medical Tribune Kinderund Jugendmedizin http://www.medical-tribune.de/verlag/produkte/ artikeldetail/fachzeitung-medical-tribune-kinder-und-jugendmedizin. html

24 Moed HF. New developments in the use of citation analysis in research evaluation. Arch Immunol Ther Exp (Warsz) 2009; 57: 13-18

25 OmniMed Verlagsges.mbH. PÄD Praktische Pädiatrie 2011; 17: 1-328

26 Paul T. The PAN study: The first step into the future. Klin Padiatr 2010; 222: 281-281

27 Prometus Verlag. Arzt + Kind www.prometus.at

28 Saleem $T$. The Hirsch index - a play on numbers or a true appraisal of academic output? Int Arch Med 2011; 4: 25 Published online 2011 July 7. doi: 10.1186/1755-7682-4-25

29 Satyanarayana $K$. Impact factor and other indices to assess science, scientists and scientific journals. Indian J Physiol Pharmacol 2010; 54: $197-212$

30 Schattauer Verlag. Kinder- und Jugendmedizin 2011; 11: 1-363

31 Schlegel N, Bardet V, Kenet G. et al. Diagnostic and therapeutic considerations on inherited platelet disorders in neonates and children. Klin Padiatr 2010; 222: 209-214

32 Schmidt B, Adelmann C, Stützer $H$ et al. Comparison of sufentanil vs. fentanyl in ventilated term neonates. Klin Padiatr 2010; 222: 62-66

33 Schmidt-Römhild-Verlag. Neuropaediatrie in Klinik und Praxis http:// neuropaediatrie-online.com/

34 ScholarOne Manuscripts http://mc.manuscriptcentral.com/klin_ padiatr

35 Springer Verlag. Monatsschr Kinderheilk 2011; 159: 1-1143. http:// www.springer.com/medicine/pediatrics/journal/112

36 Springer Verlag. Pädiatrie hautnah 2011; 23: 1-508

37 Springer Verlag. Pädiatrie \& Pädologie 2011; 46:(5) 1-32

38 Streif W, Knöfler R, Eberl W. Inherited disorders of platelet function in pediatric clinical practice: A diagnostic challenge. Klin Padiatr 2010; 222: 203-208

39 Thomson Reuters. http://science.thomsonreuters.com/m/pdfs/mgr/ jcr_0805_q.pdf

40 Thomson Reuters. http://thomsonreuters.com/products_services/ science/science_products/a-z/isi_web_of_knowledge/

41 Weinspach S, Tenenbaum T, Schönberger S et al. Cat scratch disease Heterogeneous in clinical presentation: Five unusual cases of an infection caused by Bartonella henselae. Klin Padiatr 2010; 222: 73-78

42 Weller AC. Editorial Peer Review: Its Strengths and Weaknesses. asis\&t, 2001, ISBN 1-57387-100-1 